\title{
INTESTINAL OBSTRUCTION GIVING RISE TO SYMPTOMS SIMULATING THE PAINS OF LABOUR.
}

\author{
By ALEXANDER KNOX, M.D., Physician to the Dispensary, Strangford, \\ County Down, Ireland.
}

EarLy in the summer of 1847 , an application was made to me to visit the sister of a small farmer, who was stated to be suffering from a "stoppuge", and to be in great danger, as scammony, castor oil, jalap, croton oil, with other purgatives and aperient injections, had been administered without effect. I found the patient in bed, somewhat flushed and heated, but the symptoms of acute abdominal inflammation were absent. She was, however, suffering intense agony at intervals of a few minutes, accompanied by great expulsatory efforts of the abdominal muscles, very closely resembling the regular pains of labour. The history of the case, however, as well as the character of the patient, who was not married, led to the conclusion, that I had not an uterine affection of any description to deal with-an opinion which I confirmed by examination. The abdomen was not tympanitic, but enlarged, and presenting to the touch something of a foeling of solidity. Connecting this with the expulsatory efforts before-mentioned, I was led to form the opinion, that there was obstruction arising from some solid body impacted in the alimentary canal, causing a reflex excitement of the abdominal muscles, which were thereby strongly stimulated to get rid of the source of irritation. The presence of one of those enterolithes, or intestinal concretions, so well described by Good, and more recently by the younger Monro, whose descriptions are chiefly drawn from the observations and specimens collected by his father, suggested itself. Having oiled my hand, $I$ introduced it as far as possible into the anus, for the purpose of exploration, when I detected some firm bodies completely plugging up the rectum, and succeeded in removing a number of them, some entire, and others broken down by the fingers and the shank of an iron spoon, in the process of extraction. They were of a spheroid form, and each about the size of a small apple, and of a deep grass-green colour, and firm tenacious consistence. Having hooked out as much of the entire mass as I could with my fingers, to the great and immediate relief of the patient, I administered a large emollient injection with Read's syringe, which was then perfectly effectual in unloading the lower bowels, although it had produced no effect when previously employed. A course of enemata and gentle aperients left the patient, within a week, perfectly restored to health. I naturally felt curious to ascertain the composition of these green balls, to which I had never seen anything similar. I found them, by breaking one or two of them down on a clein towel, to consist entirely of green vegetable matter, firmly agglutinated with the natural mucous excretion of the intestines. When this connecting medium was removed by frequent ablution, the entire residue was found to consist of the fibres and minute fragments of a green vegetable substance, which, on farther

1 Cyclop. Prac. Surgery, Part 1. 
examination and inquiry, I found to be that $o f$ the early cabbage, which had then come into season; and, as there were no potatoes at that time, had, together with bacon, constituted the principal part of the patient's food for several days previously. Not the slightest trace of bile was apparent in the entire mass; nor, in fact, was there anything which I conld detect, besides the vegetable matter alluded to, and the mucus, which formed the cementing medium. Denman, under the appellation of ball-stools, describod bodies similar as to form and size; but totally differing in composition, and points out the necessity of removing them by raking, as they cause extraordinary action of the intestines, and pain, periodical in its returus, and violent in a degree. In the present case, as the strongest purgatives and repeated injections had failed to gire to the peristaltic action of the bowels a sufficient stimulus for their expulsion, it is manifest that if mechanical relief had not been afforded, or had been too long delayed, a dangerous and perhaps fatal attack of inflammation must have followed. The ball-stool, according to Denman, if proper and timely means are not used, sometimes proves fatal, by bringing on a sphacelation of the parts.

Strangford, Ireland, Angust 1850 .

\section{CASE OF COMPLETE PARALYSIS OF THE MOTOR OCULI OF THE LEFT SIDE; DEPENDENT UPON ANEURISM OF THE LEFT POSTEKIOR COMMUNICATING ARTERY.}

BY CHARLES J. HARE, M.D.Cantab.; Licentiate of the Royal College of Physicians, Assistant-Physician to University College Hospital.

ANEURISYs of the cerebral arteries are of sufficiently rare occurrence to make a record of the symptoms to which they have given rise, and of the pathological appearances accompanying them, both desirable and interesting; and $\mathrm{as}$, in the case which has recently fallen under my notice, the peculiar position of the Aneurism gave rise to some pheno. mena during life, and to some circumstances in the mode of death, which, together, tended to throw light on the diagnosis of the particular lesion present, I have thought that the following details might not be unacceptable.

Case. Rosa $H$., æt. 18, unmarried, first came under my care as an out-patient at University College Hospital, April 18, 1850. By occupation a milliner, working principally at a house of business, and consequently much confined in-doors; hours late, especially during the London season, when, often, she did not get home till from 9 to 11 o'clock at night : a skilful and industrious worker; of regular habits; had sufficient food and clothing. For some time past-about a couple of years-she had resided with her mother in their present lodgings, a front room, second floor, in an apparently healthy situation ; the room is of moderate size, airy, not damp. Stature shortish ; moderately stout; conformation good; complexion rather pale; bair dark brown; pupils 